\title{
Fecal Enterotoxigenic Bacteroides fragilis- Peptostreptococcus stomatis-Parvimonas micra Biomarker for Noninvasive Diagnosis and Prognosis of Colorectal Laterally Spreading Tumor
}

\section{Xiaonan Shen}

Key Laboratory of Gastroenterology\&Hepatology, Ministry of Health; Division of Gastroenterology and Hepatology; Shanghai Institute of Digestive Disease; Renji Hospital, Shanghai Jiao Tong University School of Medicine

Jialu Li

Key Laboratory of Gastroenterology \& Hepatology, Ministry of Health; Division of Gastroenterology and Hepatology; Shanghai Institute of Digestive Disease; Renji Hospital, Shanghai Jiao Tong University School of Medicine, 145 Middle Shandong Road, Shanghai,

Jiaqi Li

Key Laboratory of Gastroenterology \& Hepatology, Ministry of Health; Division of Gastroenterology and Hepatology; Shanghai Institute of Digestive Disease; Renji Hospital, Shanghai Jiao Tong University School of Medicine, 145 Middle Shandong Road, Shanghai,

\section{Yao Zhang}

Key Laboratory of Gastroenterology \& Hepatology, Ministry of Health; Division of Gastroenterology and Hepatology; Shanghai Institute of Digestive Disease; Renji Hospital, Shanghai Jiao Tong University School of Medicine, 145 Middle Shandong Road, Shanghai,

\section{Xiaobo Li}

Key Laboratory of Gastroenterology \& Hepatology, Ministry of Health; Division of Gastroenterology and Hepatology; Shanghai Institute of Digestive Disease; Renji Hospital, Shanghai Jiao Tong University School of Medicine, 145 Middle Shandong Road, Shanghai,

\section{Qinyan Gao}

Key Laboratory of Gastroenterology \& Hepatology, Ministry of Health; Division of Gastroenterology and Hepatology; Shanghai Institute of Digestive Disease; Renji Hospital, Shanghai Jiao Tong University School of Medicine, 145 Middle Shandong Road, Shanghai,

\section{Yun Cui}

Key Laboratory of Gastroenterology \& Hepatology, Ministry of Health; Division of Gastroenterology and Hepatology; Shanghai Institute of Digestive Disease; Renji Hospital, Shanghai Jiao Tong University School of Medicine, 145 Middle Shandong Road, Shanghai,

\section{Xiaoyu Chen}


Key Laboratory of Gastroenterology \& Hepatology, Ministry of Health; Division of Gastroenterology and Hepatology; Shanghai Institute of Digestive Disease; Renji Hospital, Shanghai Jiao Tong University School of Medicine, 145 Middle Shandong Road, Shanghai,

\section{Yingxuan Chen}

Key Laboratory of Gastroenterology \& Hepatology, Ministry of Health; Division of Gastroenterology and Hepatology; Shanghai Institute of Digestive Disease; Renji Hospital, Shanghai Jiao Tong University School of Medicine, 145 Middle Shandong Road, Shanghai,

Jingyuan Fang ( $\sim$ jingyuanfang@sjtu.edu.cn )

Key Laboratory of Gastroenterology \& Hepatology, Ministry of Health; Division of Gastroenterology and Hepatology; Shanghai Institute of Digestive Disease; Renji Hospital, Shanghai Jiao Tong University School of Medicine, 145 Middle Shandong Road, Shanghai,

\section{Research}

Keywords: laterally spreading tumor, enterotoxigenic Bacteroides fragilis, Peptostreptococcus stomatis, Parvimonas micra, noninvasive biomarker, diagnosis and prognosis

Posted Date: May 21st, 2020

DOI: https://doi.org/10.21203/rs.3.rs-29514/v1

License: (1) (1) This work is licensed under a Creative Commons Attribution 4.0 International License. Read Full License 


\section{Abstract}

\section{Backgroud}

Up to now, non-invasive prediction of laterally spreading tumor (LST) and adenoma recurrence after LST resection is inevitable. The purpose of this study was to identify a microbiome signature with a high predictive effect on LSTs and a microbiome with a high predictive effect on adenoma recurrence after LSTs colectomy.

\section{Methods}

We performed 16S rRNA sequencing in mucosal samples with 5 healthy controls (HC), 8 colorectal adenoma (CRA) patients and 11 LST patients. The differentiating microbiota in fecal samples was quantified by qPCR in 475 cases including 113 HC, 208 CRA patients, 109 LST patients and 45 colorectal cancer (CRC) patients. We applied linear discriminant analysis effect size analysis to identify taxa differentially abundant between cases and controls. ROC curve was used to evaluate the diagnostic value of bacterial candidates. Pairwise comparison of AUCs was performed using the Delong's test. MantelHaenszel hazard models were used to determine the effects of microbiota composition on recurrence free survival.

\section{Results}

LST microbial dysbiosis was characterized by relative high abundance of the genus BacteroidesStreptococcus and the species enterotoxigenic Bacteroides fragilis (ETBF)-Peptostreptococcus stomatis (P. stomatis)-Parvimonas micra (P. micra). The abundance of ETBF, P. stomatis and P. micra were steadily increasing in LST and CRC groups. P.stomatis behaved stronger value on diagnosis of LST than the other two bacteria (AUC $0.887,95 \% \mathrm{Cl} 0.842-0.931$ ). The combination of P.stomatis, P.micra and ETBF (AUC $0.922,95 \% \mathrm{Cl} 0.887-0.958$ ) revealed strongest diagnostic power with $88.7 \%$ sensitivity and $81.4 \%$ specificity. ETBF, $P$. stomatis and $P$. micra were related to the malignant LST $\left(P_{P \text {. stomatis }}=0.0015, P_{P \text {. micra }}\right.$ $\left.=0.0255, P_{E T B F}=0.0169\right)$ and the abundance of IL-6. The relative high-abundance of $P$. stomatis were related to the adenoma recurrence after $\mathrm{LST}$ resection $(\mathrm{HR}=3.88, P=0.008)$.

\section{Conclusions}

Fecal microbiome signature (ETBF-P. stomatis-P. micra) can diagnose LSTs with high accuracy. ETBF, $P$. stomatis and $P$. micra were related to the malignant LST and P.stomatis exhibited a high predictive effect on the adenoma recurrence after endoscopic resection of LSTs. The signature bacteria of LST may provide a noninvasive alternative to early detect LST and predict the adenoma recurrence risk after resections.

\section{Background}


Colorectal cancers (CRC) ranked third in incidence and second in mortality among all cancers globally in 2020 (1). Almost $90 \%$ of cases of colorectal cancer develop from adenoma, especially advanced colorectal adenoma, through a series of genetic changes, known as the adenoma-carcinoma sequence, over 10 years $(2,3)$. Adenoma is the primary precursor lesion of CRC and can be removed by colonoscopic resection to avoid the carcinogenesis progress. Laterally spreading tumors (LSTs) are known for primary precursor lesion of $\mathrm{CRC}(4,5)$. LSTs are at least $10 \mathrm{~mm}$ in size and are characterized by horizontally extending growth patterns(6). It is categorized into two types: LST-Gs with nodules or granules distributed evenly or not on the surface of the lesion and LST-NGs with a smooth surface without nodules or granules(6). LST-Gs are further classified into homogeneous (LST-G-H) and nodular mixed (LST-G-M) subtypes, and LST-NGs comprise flat-elevated (LST-NG-FE) and pseudo-depressed (LST-NG-PD) subtypes. The detection rate of LST is extremely low due to the special growth pattern and morphology. Thus, there is a need to sensitively detect LSTs to prevent and reduce CRC. Moreover, adenoma recurrence after endoscopic resection of LSTs is frequent $(7,8)$. Despite the widespread use of endoscopic resection, few examined the risk factors for adenoma recurrence after LST resection. Many LST patients without adenoma recurrence are subjected to frequently and ultimately unnecessary surveillance procedures. Hence, a non-invasive, economic and convenient marker that can sensitively detect LSTs and predict the adenoma recurrence probability of LSTs yields to be explored.

The colon and rectum interact with approximately $3 \times 1013$ gut microorganisms with which the intestinal epithelium has a constant crosstalk(9). The roles of gut microbiota in the carcinogenesis colorectal cancer has received much attention in the past 5 years. Human studies generally showed that compared with healthy individuals, the gut microbiota related to CRC patients was significantly different. It showed lower abundance of potentially protective taxa, a higher species richness and increased abundance of procarcinogenic taxa (such as Porphyromonas, Escherichia, Bacteroides, Fusobacterium)(10-12). These data pinpoint a potential core set of microorganisms that might be carcinogenic. Quantifying and detecting the fold-changes in relative abundance of these bacteria forms the principle of using the microbiota as a CRC biomarker. The increasing number of studies provide a rich source for developing fecal microbial markers for CRC diagnosis. Existing studies have utilized the abundance of multiple bacterial, viral, fungal species to distinguish patients with CRC from healthy individuals and these studies exerted fairly high sensitivity and specificity(12-17). Apart from their diagnostic potential, associations between bacterial biomarkers and clinical outcomes of CRC have raised the possibility of using them as prognostic markers. Several studies have showed the relationships between tumoural amounts of bacteria in tissue with CRC survival(18-20). The microbiota may be an effective, noninvasive, economic and convenient biomarker for prognosis of CRC.

LST, an important precursor of CRC, was hardly endoscopic recognized and resected for exhibiting flat appearance. Understanding the microbiome signature of LST could give rise to new strategies to detect, reduce, or slow the progression of LST. Current noninvasive stool-based screening tests have low sensitivities for detecting LST. While microbiome signature for LSTs and noninvasive stool-based screening tests remain rare-reported. For population-wide screen and surveillance of LSTs, non-invasive, 
cost-effective and convenient biomarkers are urgently desired, since these features are obviously beneficial to the compliance of patients.

In this study, we identified a microbiome signature highly predictive of LSTs in differentiating from healthy controls and colorectal adenoma. Moreover, we identified the microbiota with high accuracy on predicting the adenoma recurrence after colonscopic resection of LSTs.

\section{Materials And Methods}

\section{Study design and participants}

This retrospective cohort was done at the Endoscopy Center, Renji Hospital, Shanghai Jiao Tong University. Approval for this study was obtained from the ethics committee of Renji Hospital, School of Medicine, Shanghai Jiao Tong University. The study complied with the Helsinki Declaration of 1975. A total number of 475 patients was consisted with 113 healthy controls, 208 CRA patients, 109 LST patients and 45 CRC patients (Work flowchart see Fig.1). To avoid potential alternation of the gut microbiota, excluding criteria were established. The exclusion criteria included: 1 ) with history of upper GI tracts surgery; 2) with a history of familial adenomatous polyposis(FAP), hereditary non-polyposis colorectal cancer(Lynch syndrome) or Peutz-Jeghers syndrome or uncontrolled diabetes, hypertension or other chronic metabolic disorder; 3) with history of uninterested gastrointestinal tract neoplasia; 4) with active $\mathrm{Gl}$ tracts bleeding in recent 6 months; 5 ) using any of the following medicine: nonsteroidal antiinflammatory drugs, immunosuppressor, antibiotics or probiotics at least 1 month prior to enrollment; 6 ) with eating habits changes in recent 4 weeks(17).

Enrolled subjects were divided into four groups: healthy control $(\mathrm{HC})$ group, CRA group, LST group, CRC group. The clinical phenotype was set by the endoscopic and pathological diagnosis. Tumors in the caecum, ascending colon, hepatic flexure, transverse colon or splenic flexure were considered to be proximal ones, while distal tumors included those in the rest of colon or rectum. Conventional-type colorectal adenomas include tubular adenoma, tubulovillous adenoma and villous adenoma. Serrated polyps include hyperplastic polyps and serrated adenoma. The early stage $\mathrm{CRC}(\mathrm{S} 0)$ as well as stage 0/pTis CRC were defined as intramucosal carcinoma and adenoma with high-grade dysplasia. Adenoma recurrence was endoscopically confirmed under 3 to 36 months' surveillance. Recurrence at each period of surveillance was defined as detection of the recurrent adenoma in patients who had no evidence of recurrence at any previous surveillance. Late recurrence was defined as adenoma at the site of previous resection at $\geq 12$ months' surveillance in patients who had no evidence of recurrent adenoma at the previous surveillance.

\section{Stool and mucosal samples collection}

All 475 stool samples (113 HC, 208 CRA patients, 109 LST patients, 45 CRC patients) were collected in the special germ-free containment before bowel preparation for endoscopy. All stool samples were moved to $-20{ }^{\circ} \mathrm{C}$ for temporary preservation and transferred to $-80{ }^{\circ} \mathrm{C}$ for long-term storage within $48 \mathrm{~h}$. All 24 
mucosal samples (5 HC, 8 CRA patients, 11 LST patients) were collected in the special germ-free containment after endoscopic resection and were moved to $-80{ }^{\circ} \mathrm{C}$ for storage immediately.

\section{DNA extraction, 16S rRNA gene sequencing and real time quantitative polymerase chain reaction}

QIAamp DNA Stool Mini Kit and QIAamp DNA Tissue Mini Kit were used according to manufacturer's instructions (Qiagen, Hilden, Germany). All extracts were preserved at $-20^{\circ} \mathrm{C}$ before $16 \mathrm{~S}$ rRNA sequencing and subsequent qPCR amplification. We performed 16S rRNA sequencing in mucosal samples. The $16 \mathrm{~S}$ rRNA V3-V4 region was amplified by PCR and the sequenced using Miseq platform (Illumia, San Diego, California, USA). The primers sequences were described in Supplementary Table 1. All primers were synthesized and purified by Sangon Biotech Shanghai. $10 \mu$ SYBR Green II was used as qPCR reaction system by TAKARA cooperation with SYBR ${ }^{\circledR}$ Premix Ex TaqTMII (TliRNaseH Plus). Stepone ${ }^{\circledR}$ plus by ABI company was used in qPCR with all the operation and configuration according to the manufacture's instruction with 40 cycles of $95^{\circ} \mathrm{C}$ denaturation for $5 \mathrm{~s}$, and $60^{\circ} \mathrm{C}$ annealing and extension for $30 \mathrm{~s}$ in total after a 30 s of pre-denaturation at $95^{\circ} \mathrm{C}$.

\section{Statistical analysis}

The 16S rRNA sequencing data were analysed using Quantitative Insights Into Microbial Ecology (QIIME2 V.2018.2). DADA2 software, wrapped in QIIME2, was used to filter the sequencing reads and construct feature table. We applied linear discriminant analysis effect size (LEfSe) analysis to identify taxa differentially abundant between cases and controls. This method first uses the non-parametric factorial Kruskal-Wallis sum-rank test to detect features with significant differential abundance and then uses linear discriminant analysis (LDA) to calculate the effect size of each feature. Other statistical analysis included Fisher's exact test, Kruskal-Wallis test and partial Spearman's rank correlation (PResiduals package).

All qPCR samples were in triplicates. Average Ct value from the triplicates was calculated and the relative abundance of the target gut microbiota was based on the $\Delta \mathrm{Ct}$ value defined as the target $\mathrm{Ct}$ value subtracted $\mathrm{Ct}$ value for $16 \mathrm{~S}$ rRNA, which means the all the relative abundance was normalized by the abundance of $16 \mathrm{~S}$ rRNA(21). As for statistical analysis, values were all expressed as mean \pm SD. MannWhitney $\mathrm{U}$ test and Kruskal-Wallis test were used in comparison for continuous and nonparametric analyses respectively. Receiver Operating Characteristic (ROC) curve was used to evaluate the diagnostic value of bacterial candidates in distinguishing LST and CRA and to determine the best cut-off values that maximized the Youden index ( $\mathrm{J}=$ Sensitivity+Specificity-1). Pairwise comparison of areas under ROC (AUCs) was performed using the Delong's test. Recurrence-free survival (RFS) was defined as the time from diagnosis to recurrence of colorectal adenoma in 3 years. Patients who did not experience recurrence were censored at date of last follow-up. Mantel-Haenszel hazard models were used to determine the effects of microbiota composition on RFS. All tests were done by SPSS statistical software (version 23.0, IBM Corp, Armonk, NY, 2012) and R statistical software (version 3.6.0, 2019). 


\section{Results}

\section{Bacteria composition was significantly different between LSTs and controls}

A total number of 475 patients were divided into 113 healthy controls, 208 CRA patients, 109 LST patients and 45 CRC patients (basic demographic characteristics see Supplementary Table 2; Work flowchart see Fig.1). To examine the bacteria composition in LSTs, The 16S rRNA sequencing was performed in 24 mucosal samples ( 5 HC, 8 CRA patients, 11 LST patients). Bacteria compositions in HC, CRA patients and LST patients were compared. In typing analysis, it was found that bacteria were significantly different in family, genus and species levels among the above three groups (Fig.2a). Bacteroides and Streptococcus were found to be abundant in LST group compared to the other two groups (Fig.2b-e). The potential differential bacterium patterns among the three groups was defined by Kruskal-Wallis test and LEfSe algorithm. Lactobacillus johnsonil and Bacteroided fragilis were significantly abundant in LST group (Fig.3a,b). By analyzing the GMrepo bacteria database, the abundance of Peptostreptococcus stomatis and Parvimonas micra were found to be postively relevant to the abundance of Bacteroided fragilis (Fig.3c). Moreover, enterotoxigenic Bacteroides fragilis(ETBF) was reported to be driver bacteria in colorectal cancer development.

\section{The abundance of ETBF, P. stomatis and P. micra were increased in LST and CRC groups}

$E T B F$, P. stomatis, P. micra and Lactobacillus johnsonil were also tested in fecal samples. An increase in the relative abundance of ETBF, P. stomatis and P. micra in LST patients over $\mathrm{HC}$ and CRA patients was observed. Moreover, there was an increase of the above microbiota in CRC patients over LST patients, as well (Figure 4A). Comparing LST patients with healthy controls, ETBF increased by 9.26 folds $(P<$ $0.0001)$, $P$. stomatis increased by 14.79 folds $(P<0.0001)$ and $P$. micra increased by 10.17 folds $(P<$ 0.0001). Comparing CRA patients with LST patients, ETBF increased by 2.95 folds $(P<0.0001), P$. stomatis increased by 8.76 folds $(P<0.0001)$ and $P$. micra increased by 6.65 folds $(P<0.0001)$. Comparing LST patients with CRC patients, ETBF increased by 3.00 folds $(P=0.0327), P$. stomatis increased by 15.31 folds $(P=0.0058)$ and $P$. micra increased by 6.41 folds $(P=0.0413)$. Further, no discrepancy was found for the relative abundance of three intestinal bacteria among distal and proximal colon for LSTs (Supplementary Figure 1A). In terms of topography and histopathology, no distinction showed between LST-G and LST-NG, as well as traditional adenoma type and serrated polyp type (Supplementary Fig. 1b,c). In addition, the dysplasia degree led to totally different clinical decision and prognosis. Accordingly, the three bacteria in terms of dysplasia degree reported by pathologist were also tested. The SO LST had higher abundance of fecal bacteria $\left(P_{P \text {. stomatis }}=0.0015, P_{P \text {. micra }}=0.0255, P_{E T B F}\right.$ $=0.0169$ ) (Fig.4b). The inflammatory cytokines (IL-1 $\beta$, IL-6, IL-8, IL-10, TNF-a) data of LST patients further showed that $P$. stomatis, $P$. micra and ETBF significantly increased IL-6 secretion (Fig.4C) and the abundance of P.stomatis $\left(P_{P \text {. stomatis }}=0.0024\right)$ and P.micra $\left(P_{P \text {. micra }}=0.0077\right)$ positively correlated with the abundance of $\operatorname{ETBF}$ (Fig.4d). 
As for Lactobacillus johnsonil, no significant changes have been revealed among CRA and LST groups (Supplementary Fig.2). Therefore, ETBF, P. stomatis and P. micra were selected as non-invasive candidate biomarkers for detecting LSTs.

\section{The predictive values of ETBF, P. stomatis and $P$. micra for the occurrence of LSTs}

The relative abundance of the ETBF-P.stomatis-P.micra microbiome showed an increasing in the LST group as compared to HC group and CRA group as described above (Fig.4a). In comparison between HC group and LST group, the ROC curves were performed. In terms of AUCs, P.stomatis behaved stronger than the other two bacteria ( $\left.\mathrm{AUC}_{\text {P.stomatis }} 0.887,95 \% \mathrm{Cl} 0.842-0.931\right)$ (Fig.5a). These three contributing markers were therefore united as an independent model. Combination of P.stomatis-P.micra and P.stomatis-P.micra-ETBF revealed stronger diagnostic potential over the single P.stomatis $\left(P_{P . \text { stomatis-P.micra }}\right.$ $\left.=0.0376, P_{\text {P.stomatis-P.micra-ETBF }}=0.00930\right)($ Fig.5b). The combination of the three bacteria showed the maximum AUC (AUC $0.922,95 \% \mathrm{Cl} 0.887-0.958$ ) and Youden index with $88.7 \%$ sensitivity and $81.4 \%$ specificity (Table 1$)$.

Table 1. AUC and diagnostic value of different biomarkers for predicting LST

\begin{tabular}{|c|c|c|c|c|c|c|c|}
\hline \multirow{2}{*}{ Detection LST from HC } & \multirow[b]{2}{*}{ ETBF } & AUC & \multicolumn{2}{|c|}{$95 \% \mathrm{CI}$} & Sensitivity & Specificity & \multirow{2}{*}{$\begin{array}{c}\text { Youden Index } \\
0.549\end{array}$} \\
\hline & & 0.795 & 0.735 & 0.855 & 0.752 & 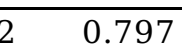 & \\
\hline & Ps & 0.887 & 0.842 & 0.931 & 0.936 & 0.726 & 0.661 \\
\hline & $\mathrm{Pm}$ & 0.842 & 0.790 & 0.893 & 0.706 & 0.850 & 0.556 \\
\hline & ETBF+Ps & 0.904 & 0.864 & 0.944 & 0.899 & 0.788 & 0.687 \\
\hline & $\mathrm{ETBF}+\mathrm{Pm}$ & 0.880 & 0.835 & 0.925 & 0.844 & 0.823 & 0.667 \\
\hline & $\mathrm{Ps}+\mathrm{Pm}$ & 0.909 & 0.871 & 0.947 & 0.862 & 0.814 & 0.677 \\
\hline & $\mathrm{ETBF}+\mathrm{Ps}+\mathrm{Pm}$ & 0.922 & 0.887 & 0.958 & 0.881 & 0.814 & 0.695 \\
\hline \multirow[t]{7}{*}{ Detection LST from CRA } & ETBF & 0.645 & 0.584 & 0.707 & 0.725 & 0.620 & 0.345 \\
\hline & Ps & 0.807 & 0.760 & 0.854 & 0.844 & 0.678 & 0.522 \\
\hline & $\mathrm{Pm}$ & 0.787 & 0.735 & 0.840 & 0.706 & 0.769 & 0.476 \\
\hline & ETBF+Ps & 0.808 & 0.761 & 0.855 & 0.835 & 0.688 & 0.522 \\
\hline & $\mathrm{ETBF}+\mathrm{Pm}$ & 0.791 & 0.739 & 0.842 & 0.633 & 0.841 & 0.474 \\
\hline & $\mathrm{Ps}+\mathrm{Pm}$ & 0.835 & 0.790 & 0.879 & 0.762 & 0.808 & 0.569 \\
\hline & ETBF+Ps+Pm & 0.835 & 0.790 & 0.879 & 0.762 & 0.808 & 0.569 \\
\hline
\end{tabular}

$\mathrm{HC}=$ Healthy control. $\mathrm{CRA}=$ Colorectal adenoma. $\mathrm{LST}=$ Laterally spreading tumor. $E T B F=$ Enterotoxigenic Bacteroides fragilis. Ps= Peptostreptococcus stomatis. Pm= Parvimonas micra. To distinguish LSTs from CRAs, the ROC curves were made. The result showet that P.stomatis exhibited higher AUCs than the other two bacteria ( $\mathrm{AUC}_{\text {P.stomatis }} 0.807,95 \% \mathrm{Cl} 0.760-0.854$ ) (Fig.5c). Nonetheless, the diagnostic combination of P.stomatis-P.micra and P.stomatis-P.micra-ETBF showed higher AUCs than the single P.stomatis $\left(P_{\text {P.stomatis-P.micra }}=0.0411, P_{\text {P.stomatis-P.micra-ETBF }}=0.0416\right)$ (Figure $\left.5 D\right)$. In terms of Youden index, the P.stomatis-P.micra marker was equal to the P.stomatis-P.micra-ETBF marker with $76.2 \%$ sensitivity and $80.8 \%$ specificity (Table 1 ). 


\section{P. stomatis were positively related to adenoma recurrence after resection of LSTs}

Considering the relationship between LST signature bacteria and tumoral development, the next step of this study aims to determine if there were differences in the above three bacteria composition between recurrence or non-recurrence adenoma after resection of LSTs. After 36 months' follow-ups, there were 23 LST patients with adenoma recurrence among 92 LST patients. To this end, the distribution of ETBF, $P$. stomatis and $P$. micra among the recurrence group and non-recurrence group was first evaluated. The recurrence group exhibited a predominant abundance of $P$. stomatis $(P<0.0001)$. Besides, no difference of the other two bacteria between the two groups were detected (Fig.6a) and $P$. stomatis presented strong value on predicting the adenoma recurrence (AUC $0.800,95 \% \mathrm{Cl} 0.678-0.915, P<0.0001$ ). Based on these results, the enrolled patients were divided into high abundance of $P$. stomatis group and low abunance of $P$. stomatis group. Univariate analysis showed the dysplasia type of LST and the abundance of $P$. stomatis were related to the adenoma recurrence, while gender, age, history of hypertension or diabetes mellitus, size, location, topography, histopathology futiled (Supplementary Table 3 ). The survival curve showed the SO LSTs (HR = 2.78, $P=0.026)$ and high-abundance of $P$. stomatis $(\mathrm{HR}=3.88, P=0.008)$ were related to the adenoma recurrence event (Fig.6b). Furthermore, the abundance difference of P.stomatis in early-recurrence group and late-recurrence group was also tested to be no significant difference between the above two groups (Supplementary Fig.3).

\section{Discussion}

There is growing evidence that alteration in the intestinal microbiome correlate with colorectal neoplasms $(12,22,23)$. However, LST, as a principal precursor lesion of CRC, was never reported about the intestinal microbiota signature. Moreover, early detection and predicting the risk of adenoma recurrence after LST resection were essential to prevent CRC. Herein, the gut microbiota structure of microbiome in LST cohort by means of $16 \mathrm{~S}$ rRNA gene sequencing was delineated in this study. The results demonstrate that LST microbial dysbiosis was characterized by relative high abundance of the genus Bacteroides-Streptococcus and the species ETBF-P. stomatis-P. micra. Based on the microbial signature, fecal microbiobial biomarkers ETBF-P. stomatis-P. micra were defined as early detection of LST. It shows that disease-associated bacteria may form a synergistic network which result in diseases. Moreover, $P$. stomatis behaved high accuracy on predicting adenoma recurrence after LST resections.

ETBF has been known as a contributor to chronic colonic diseases, including oncogenic transformation, intestinal inflammation, chronic colonic dysfunctions and colorectal precancerous and cancerous lesions(24-28). Combined action of the ETBF and IL-17 on colonic epithelial cells promoted the differentiation of monocytic-myeloid-derived suppressor cells(MO-MDSCs) and suppressed T-cell proliferation(27). Bacteroides fragilis-associated IncRNA1 (BFAL1) is a mediator of ETBF-induced carcinogenesis and may be a potential therapeutic target for ETBF-induced CRC(28). The recent observation of ETBF-bearing biofilms in colon biopsies from humans with colon cancer susceptibility loci strongly suggests that ETBF plays one key role in colorectal cancer(29). P. stomatis and P.micra are gram-

positive anaerobic bacterium and part of the oral and gut commensal flora $(30,31)$. The organisms have 
been implicated as a causative agent of several diseases(12, 13, 30,32-34), including oral squamous cell carcinoma(32) and apical abscess(33). A meta-analysis of 526 fecal shotgun metagenome datasets identified a microbial core of seven enriched bacteria in CRC(22). This core set included: ETBF; four oral bacteria of Fusobacterium nucleatum(35,36), P.micra, Prevotella intermedia(37) and Porphyromonas asaccharolytica(38); and two other bacteria, Thermanaerovibrio acidaminovorans(22) and Alistipes finegoldii(39). Yachida et al(23) collected stool samples from 616 participants and found these bacteria were apparent in cases of multiple colorectal adenomas and intramucosal carcinomas. Stage-specific analyses showed two patterns of significant species elevation: the first increased across early to later stages, whereas the second was elevated only in the early stages. The former was characterized by $F$. nucleatum, Solobacterium moorei(37), P.stomatis, Peptostreptococcus anaerobius(40, 41), Lactobacillus sanfranciscensis, P.micra and Gemella morbillorum(42). ETBF, P.stomatis and P.micra were crucial to colorectal carcinogenesis while they were rarely reported to be biomarkers to detect precancerous lesions.

Several studies have utilized the abundance of multiple bacterial species to distinguish patients with CRC from healthy individuals. Among several bacterial candidates, F. nucleatum emerged as a key marker either when being quantified alone $(16,21)$ or combined with other bacteria $(13,17,43)$, specifically Clostridium symbiosum(17). These findings provide support for an accurate stool-based diagnostic test using markers targeting a limited number of microbial species. In this study, the fecal abundance of the three bacteria ETBF-P. stomatis-P. micra displayed considerably high sensitivity and specialty in detecting LST, especially P. stomatis. While combinations of P.stomatis-P.micra-ETBF revealed higher diagnostic potential compared with the single biomarker P.stomatis and combined biomarkers of P.stomatis-P.micra, P.stomatis-ETBF, P.micra-ETBF. The wide application of the above bacterial markers in LST detection could be rather feasible since qPCR detection of fecal bacterial DNA in stool is technically more reliable and cost-effective than endoscopic screening.

Adenoma recurrence after endoscopic resection of LSTs is frequent $(7,8,44,45)$. Despite the widespread use of endoscopic resection, few examined the risk factors for recurrence. LSTs with cancerous histology were reported associated with the adenoma recurrence after endoscopic therapy(46). Some studies showed that independent risk factors recurrent adenoma were piecemeal resection, adjunctive argon plasma coagulation, lesion occupying $75 \%$ of the luminal circumference, lesion size $>60 \mathrm{~mm}$, and high grade dysplasia of $\operatorname{LSTs}(8,46,47)$. Furthermore, adenoma recurrence surveillance requests regular endoscopy screening. Many patients not developing adenoma recurrence have to subjected to frequently and ultimately unnecessary surveillance procedures. Carefully installed devices, well-trained technician and inconvenient bowel preparation before endoscopy are resource-consuming and may limit the use for population-wide screening. Based on this study, it is worth noting that the relative high abundance of P.stomatis positively correlated with adenoma recurrence after LST resection. Application of the biomarker P.stomatis to predict adenoma recurrence was therefore promising and feasible.

Furthermore, ETBF-P. stomatis-P. micra modulation, especially P. stomatis may be important for LST development, adenoma recurrence after LST resection and even colorectal carcinogenesis. Efforts need 
to find the best interventional approaches to manipulate the microbiota. It will necessitate concerted efforts from researchers and industrial partners to translate it into a clinical product.

However, there is no clear answer about the role of ETBF-P. stomatis-P. micra in LST or in CRC. The process is intricate and influenced by genetic and environmental factors. Mechanisms include immune regulation, metabolism of dietary components, genotoxin production and inflammation(48-50). There remained a question what are the driver and passenger bacteria in LST development? As described above, the three bacteria increased IL- 6 secretion in LST group. It can be hypothesized that the three bacteria induce inflammatory environment to accelerate the development of LSTs. While it deserves indepth studies about carcinogenesis as well as cancer therapy responses.

The strengths of the present study were as follows.First, we reported for the first time that the bacteria signature of LST. Second, we found fecal microbiome signature (ETBF-P. stomatis -P. micra) can effectively predict the occurence of LSTs. Moreover, ETBF, $P$. stomatis and $P$. micra were related to the SO stage LST. Third, our study provided new evidence that P.stomatis exhibited the high accuracy on predicting the adenoma recurrence after endoscopic resection of LSTs.

Although clinical data for endoscopic resections was collected prospectively and all consecutive patients were included, this study was limited by its retrospective design, as all these results were from a single tertiary center and may not be generalizable. In future, studies in large cohorts enrolling more patients need to be carried out, which can derive the best diagnostic algorithm across populations.

\section{Conclusion}

In conclusion, we observed, for the first time, fecal microbiome signature (ETBF-P. stomatis -P. micra) can effectively predict the presence of LSTs. Moreover, ETBF, P. stomatis and $P$. micra were related to malignant LST and P.stomatis exhibited the high accuracy on predicting the adenoma recurrence after endoscopic resection of LSTs. Thus, the signature bacteria of LST can provide a noninvasive method to early detect LST and predict the adenoma recurrence risk after resections.

\section{Abbreviations}

LST: Laterally spreading tumor

HC: Healthy control

CRA: Colorectal adenoma

CRC: Colorectal cancer

ETBF: Enterotoxigenic Bacteroides fragilis

P. stomatis: Peptostreptococcus stomatis

Page $11 / 22$ 
LEfSe: Linear discriminant analysis effect size

LDA: Linear discriminant analysis

qPCR: Real time quantitative polymerase chain reaction

ROC: Receiver operating characteristic

AUC: Areas under receiver operating characteristic

RFS: Recurrence-free survival

\section{Declarations}

\section{Ethics approval and consent to participate}

This study has been conducted in accordance with ethical standards and according to the Helsinki Declaration of 1975. The study protocol was approved by the ethics committee of Renji Hospital, School of Medicine, Shanghai Jiao Tong University.

\section{Consent for publication}

Not applicable.

\section{Availability of data and materials}

The datasets used and/or analyzed during the current study are available from the corresponding author on reasonable request.

\section{Competing Interests}

The authors declare that there are no competing interests.

\section{Funding}

This work was supported by grants from the National Natural Science Foundation of China (81421001, 81530072, 81830081).

\section{Authors' Contributions}

Xiaonan Shen analysed the data and wrote the manuscript. Xiaonan Shen, Jialu Li, Jiaqi Li, Yao Zhang, Xiaobo Li, Qinyan Gao conceived the study and collected samples. Yun Cui and Xiaoyu Chen performed the pathological diagnosis. Yingxuan Chen and Jingyuan Fang revised the manuscript. Jingyuan Fang designed and supervised the study. All authors read and approved the final manuscript. 


\section{Acknowledgements}

We are grateful to all members of the group at the Shanghai Institute of Digesitve Disease.

\section{References}

1. Siegel RL, Miller KD, Jemal A. Cancer statistics, 2020. CA Cancer J Clin. 2020;70(1):7-30.

2. Cotton S, Sharp L, Little J. The adenoma-carcinoma sequence and prospects for the prevention of colorectal neoplasia. Crit Rev Oncog. 1996;7(5-6):293-342.

3. Lee-Six H, Olafsson S, Ellis P, Osborne RJ, Sanders MA, Moore L, et al. The landscape of somatic mutation in normal colorectal epithelial cells. Nature. 2019;574(7779):532-7.

4. Ishigaki T, Kudo SE, Miyachi H, Hayashi T, Minegishi Y, Toyoshima N, et al. Treatment policy for colonic laterally spreading tumors based on each clinicopathological feature of 4 subtypes: actual status of pseudo-depressed type. Gastrointest Endosc. 2020.

5. Kumarasinghe MP, Bourke MJ, Brown I, Draganov PV, McLeod D, Streutker C, et al. Pathological assessment of endoscopic resections of the gastrointestinal tract: a comprehensive clinicopathologic review. Mod Pathol. 2020.

6. Kudo S. Endoscopic mucosal resection of flat and depressed types of early colorectal cancer. Endoscopy. 1993;25(7):455-61.

7. Hassan C, Repici A, Sharma P, Correale L, Zullo A, Bretthauer M, et al. Efficacy and safety of endoscopic resection of large colorectal polyps: a systematic review and meta-analysis. Gut. 2016;65(5):806-20.

8. Moss A, Williams SJ, Hourigan LF, Brown G, Tam W, Singh R, et al. Long-term adenoma recurrence following wide-field endoscopic mucosal resection (WF-EMR) for advanced colonic mucosal neoplasia is infrequent: results and risk factors in 1000 cases from the Australian Colonic EMR (ACE) study. Gut. 2015;64(1):57-65.

9. Qin J, Li R, Raes J, Arumugam M, Burgdorf KS, Manichanh C, et al. A human gut microbial gene catalogue established by metagenomic sequencing. Nature. 2010;464(7285):59-65.

10. Castellarin M, Warren RL, Freeman JD, Dreolini L, Krzywinski M, Strauss J, et al. Fusobacterium nucleatum infection is prevalent in human colorectal carcinoma. Genome Res. 2012;22(2):299-306.

11. Feng Q, Liang S, Jia H, Stadlmayr A, Tang L, Lan Z, et al. Gut microbiome development along the colorectal adenoma-carcinoma sequence. Nat Commun. 2015;6:6528.

12. Yu J, Feng Q, Wong SH, Zhang D, Liang QY, Qin Y, et al. Metagenomic analysis of faecal microbiome as a tool towards targeted non-invasive biomarkers for colorectal cancer. Gut. 2017;66(1):70-8.

13. Baxter NT, Ruffin MTt, Rogers MA, Schloss PD. Microbiota-based model improves the sensitivity of fecal immunochemical test for detecting colonic lesions. Genome Med. 2016;8(1):37.

14. Nakatsu G, Zhou H, Wu WKK, Wong SH, Coker OO, Dai Z, et al. Alterations in Enteric Virome Are Associated With Colorectal Cancer and Survival Outcomes. Gastroenterology. 2018;155(2):529-41 e5. 
15. Gao R, Kong C, Li H, Huang L, Qu X, Qin N, et al. Dysbiosis signature of mycobiota in colon polyp and colorectal cancer. Eur J Clin Microbiol Infect Dis. 2017;36(12):2457-68.

16. Suehiro Y, Zhang Y, Hashimoto S, Takami T, Higaki S, Shindo Y, et al. Highly sensitive faecal DNA testing of TWIST1 methylation in combination with faecal immunochemical test for haemoglobin is a promising marker for detection of colorectal neoplasia. Ann Clin Biochem. 2018;55(1):59-68.

17. Xie YH, Gao QY, Cai GX, Sun XM, Zou TH, Chen HM, et al. Fecal Clostridium symbiosum for Noninvasive Detection of Early and Advanced Colorectal Cancer: Test and Validation Studies. EBioMedicine. 2017;25:32-40.

18. Mima K, Nishihara R, Qian ZR, Cao Y, Sukawa Y, Nowak JA, et al. Fusobacterium nucleatum in colorectal carcinoma tissue and patient prognosis. Gut. 2016;65(12):1973-80.

19. Wei Z, Cao S, Liu S, Yao Z, Sun T, Li Y, et al. Could gut microbiota serve as prognostic biomarker associated with colorectal cancer patients' survival? A pilot study on relevant mechanism. Oncotarget. 2016;7(29):46158-72.

20. Flanagan L, Schmid J, Ebert M, Soucek P, Kunicka T, Liska V, et al. Fusobacterium nucleatum associates with stages of colorectal neoplasia development, colorectal cancer and disease outcome. Eur J Clin Microbiol Infect Dis. 2014;33(8):1381-90.

21. Wong SH, Kwong TNY, Chow TC, Luk AKC, Dai RZW, Nakatsu G, et al. Quantitation of faecal Fusobacterium improves faecal immunochemical test in detecting advanced colorectal neoplasia. Gut. 2017;66(8):1441-8.

22. Dai Z, Coker OO, Nakatsu G, Wu WKK, Zhao L, Chen Z, et al. Multi-cohort analysis of colorectal cancer metagenome identified altered bacteria across populations and universal bacterial markers. Microbiome. 2018;6(1):70.

23. Yachida S, Mizutani S, Shiroma H, Shiba S, Nakajima T, Sakamoto T, et al. Metagenomic and metabolomic analyses reveal distinct stage-specific phenotypes of the gut microbiota in colorectal cancer. Nature medicine. 2019;25(6):968-76.

24. Zamani S, Taslimi R, Sarabi A, Jasemi S, Sechi LA, Feizabadi MM. Enterotoxigenic Bacteroides fragilis: A Possible Etiological Candidate for Bacterially-Induced Colorectal Precancerous and Cancerous Lesions. Front Cell Infect Microbiol. 2019;9:449.

25. Geis AL, Fan H, Wu X, Wu S, Huso DL, Wolfe JL, et al. Regulatory T-cell Response to Enterotoxigenic Bacteroides fragilis Colonization Triggers IL17-Dependent Colon Carcinogenesis. Cancer Discov. 2015;5(10):1098-109.

26. Chung L, Thiele Orberg E, Geis AL, Chan JL, Fu K, DeStefano Shields CE, et al. Bacteroides fragilis Toxin Coordinates a Pro-carcinogenic Inflammatory Cascade via Targeting of Colonic Epithelial Cells. Cell Host Microbe. 2018;23(2):203-14 e5.

27. Thiele Orberg E, Fan H, Tam AJ, Dejea CM, Destefano Shields CE, Wu S, et al. The myeloid immune signature of enterotoxigenic Bacteroides fragilis-induced murine colon tumorigenesis. Mucosal Immunol. 2017;10(2):421-33. 
28. Bao Y, Tang J, Qian Y, Sun T, Chen H, Chen Z, et al. Long noncoding RNA BFAL1 mediates enterotoxigenic Bacteroides fragilis-related carcinogenesis in colorectal cancer via the RHEB/mTOR pathway. Cell Death Dis. 2019;10(9):675.

29. Dejea CM, Fathi P, Craig JM, Boleij A, Taddese R, Geis AL, et al. Patients with familial adenomatous polyposis harbor colonic biofilms containing tumorigenic bacteria. Science. 2018;359(6375):592-7.

30. Kistler JO, Pesaro M, Wade WG. Development and pyrosequencing analysis of an in-vitro oral biofilm model. BMC Microbiol. 2015;15:24.

31. Shah MS, DeSantis TZ, Weinmaier T, McMurdie PJ, Cope JL, Altrichter A, et al. Leveraging sequencebased faecal microbial community survey data to identify a composite biomarker for colorectal cancer. Gut. 2018;67(5):882-91.

32. Zhang L, Liu Y, Zheng HJ, Zhang CP. The Oral Microbiota May Have Influence on Oral Cancer. Front Cell Infect Microbiol. 2019;9:476.

33. Nobrega LM, Montagner F, Ribeiro AC, Mayer MA, Gomes BP. Molecular Identification of Cultivable Bacteria From Infected Root Canals Associated With Acute Apical Abscess. Braz Dent J. 2016;27(3):318-24.

34. Drewes JL, White JR, Dejea CM, Fathi P, lyadorai T, Vadivelu J, et al. High-resolution bacterial $16 \mathrm{~S}$ rRNA gene profile meta-analysis and biofilm status reveal common colorectal cancer consortia. NPJ Biofilms Microbiomes. 2017;3:34.

35. Brennan CA, Garrett WS. Fusobacterium nucleatum - symbiont, opportunist and oncobacterium. Nat Rev Microbiol. 2019;17(3):156-66.

36. Bullman S, Pedamallu CS, Sicinska E, Clancy TE, Zhang X, Cai D, et al. Analysis of Fusobacterium persistence and antibiotic response in colorectal cancer. Science. 2017;358(6369):1443-8.

37. Yang Y, Cai Q, Shu XO, Steinwandel MD, Blot WJ, Zheng W, et al. Prospective study of oral microbiome and colorectal cancer risk in low-income and African American populations. Int $\mathrm{J}$ Cancer. 2019;144(10):2381-9.

38. Ai D, Pan H, Han R, Li X, Liu G, Xia LC. Using Decision Tree Aggregation with Random Forest Model to Identify Gut Microbes Associated with Colorectal Cancer. Genes (Basel). 2019;10(2).

39. Yang $Y$, Jobin C. Novel insights into microbiome in colitis and colorectal cancer. Curr Opin Gastroenterol. 2017;33(6):422-7.

40. Long X, Wong CC, Tong L, Chu ESH, Ho Szeto C, Go MYY, et al. Peptostreptococcus anaerobius promotes colorectal carcinogenesis and modulates tumour immunity. Nat Microbiol. 2019;4(12):2319-30.

41. Tsoi H, Chu ESH, Zhang X, Sheng J, Nakatsu G, Ng SC, et al. Peptostreptococcus anaerobius Induces Intracellular Cholesterol Biosynthesis in Colon Cells to Induce Proliferation and Causes Dysplasia in Mice. Gastroenterology. 2017;152(6):1419-33 e5.

42. Kwong TNY, Wang X, Nakatsu G, Chow TC, Tipoe T, Dai RZW, et al. Association Between Bacteremia From Specific Microbes and Subsequent Diagnosis of Colorectal Cancer. Gastroenterology. 2018;155(2):383-90 e8. 
43. Guo S, Li L, Xu B, Li M, Zeng Q, Xiao H, et al. A Simple and Novel Fecal Biomarker for Colorectal Cancer: Ratio of Fusobacterium Nucleatum to Probiotics Populations, Based on Their Antagonistic Effect. Clin Chem. 2018;64(9):1327-37.

44. Li Y, Zhang Y, Chen Y, Wang Y, Dou L, Wang X, et al. Long-term outcomes of endoscopic treatment for colorectal laterally spreading tumor: a large-scale multicenter retrospective study from China. Surg Endosc. 2020.

45. Soliman H, Brieau B, Guillaumot MA, Leblanc S, Barret M, Camus M, et al. Invasive pit pattern, macronodule and depression are predictive factors of submucosal invasion in colorectal laterally spreading tumours from a Western population. United European Gastroenterol J. 2018;6(10):156977.

46. Tate DJ, Desomer L, Klein A, Brown G, Hourigan LF, Lee EY, et al. Adenoma recurrence after piecemeal colonic EMR is predictable: the Sydney EMR recurrence tool. Gastrointest Endosc. 2017;85(3):647-56 e6.

47. Emmanuel A, Lapa C, Ghosh A, Gulati S, Burt M, Hayee B, et al. Risk factors for early and late adenoma recurrence after advanced colorectal endoscopic resection at an expert Western center. Gastrointest Endosc. 2019;90(1):127-36.

48. Lasry A, Zinger A, Ben-Neriah Y. Inflammatory networks underlying colorectal cancer. Nat Immunol. 2016;17(3):230-40.

49. Zhang FF, Cudhea F, Shan Z, Michaud DS, Imamura F, Eom H, et al. Preventable Cancer Burden Associated With Poor Diet in the United States. JNCI Cancer Spectr. 2019;3(2):pkz034.

50. Tilg H, Adolph TE, Gerner RR, Moschen AR. The Intestinal Microbiota in Colorectal Cancer. Cancer Cell. 2018;33(6):954-64.

\section{Figures}


510 subjects enrolled and fecal samples acquired according to the exclusion criteria

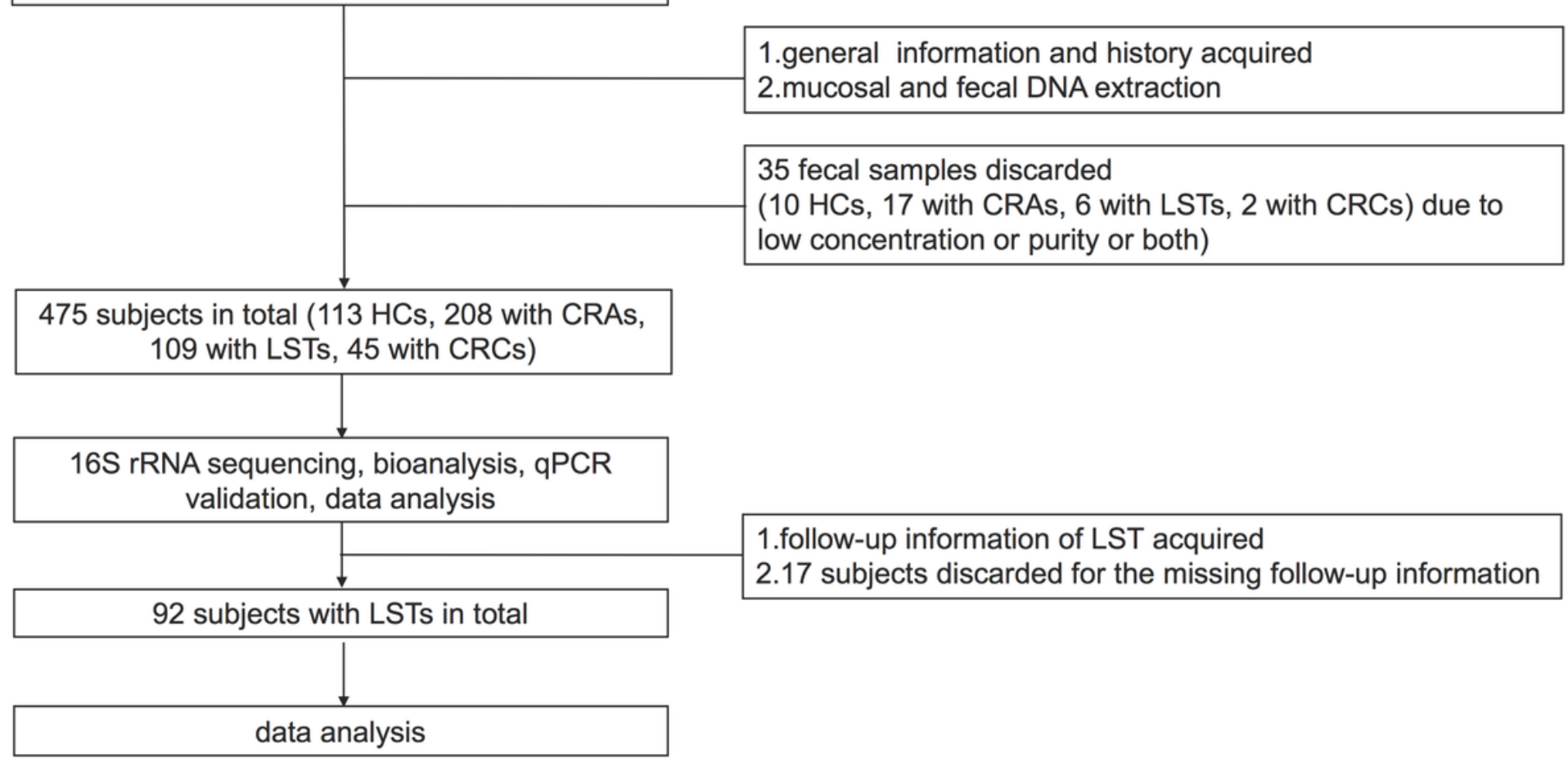

\section{Figure 1}

Work flowcharts. $\mathrm{HC}=$ healthy control. $\mathrm{CRA}=$ colorectal adenoma. $\mathrm{LST}=$ laterally spreading tumor. $\mathrm{CRC}=$ colorectal carcinoma. $\mathrm{qPCR}=$ real time quantitative polymerase chain reaction.

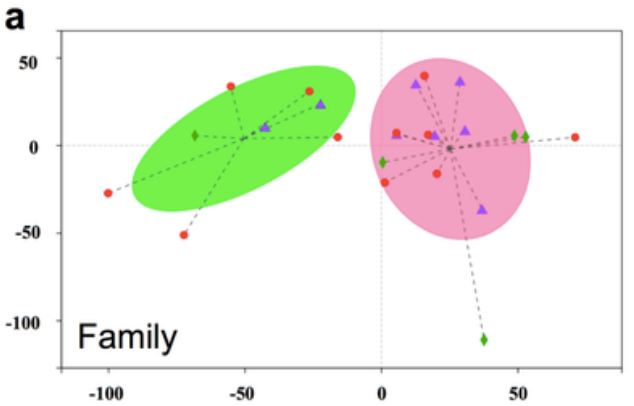

b

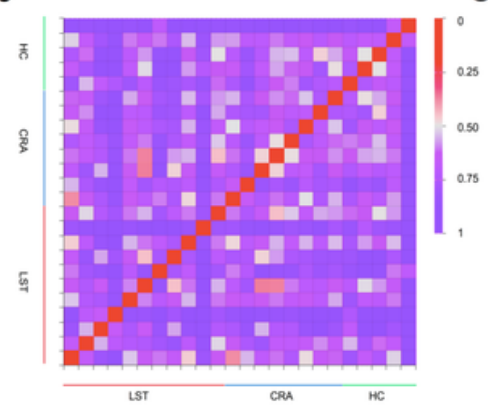

C

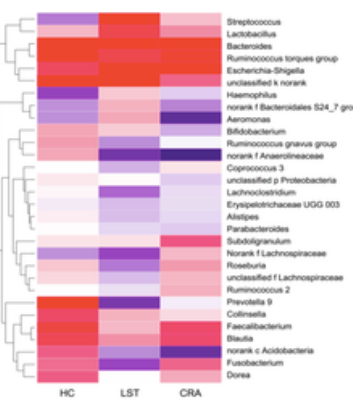

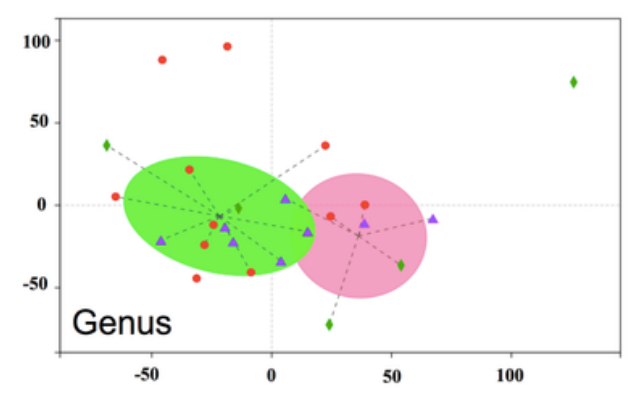

d

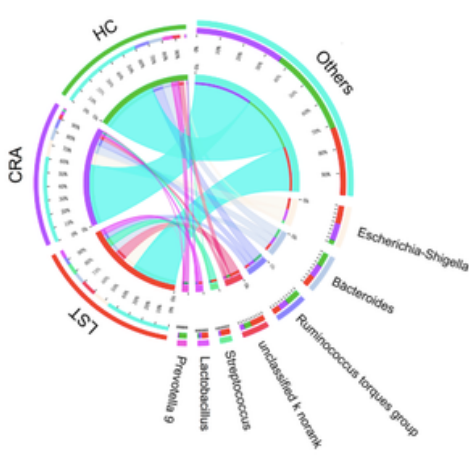

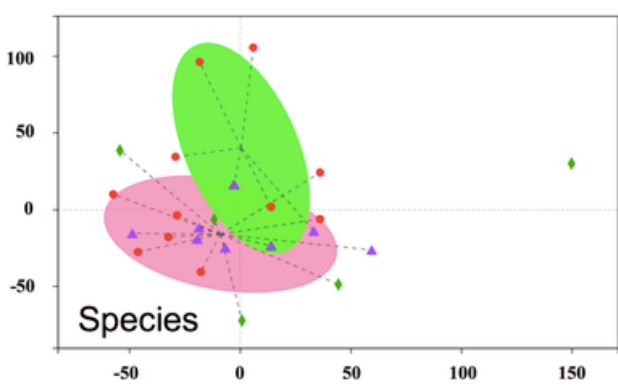

e

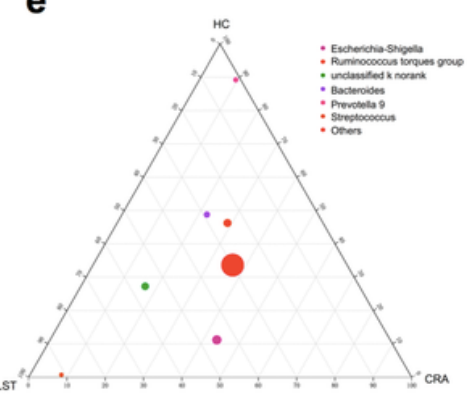

Figure 2 
Variations of mucosal microbiota composition in LST. a Typing analysis on family, genus and species groups in the $\mathrm{HC}(\mathrm{n}=5), \mathrm{CRA}(\mathrm{n}=8)$ and LST group $(n=11)$. b Distance heatmap in samples on genus level. $c$ Heatmap in the three groups on genus level, Bacteriodes and Streptococcus were significantly over presented in LST group. d Cicros analysis on genus in three groups. e Tertiary analysis on genus level in three groups. $\mathrm{HC}=$ healthy control. $\mathrm{CRA}=$ colorectal adenoma. $\mathrm{LST}=$ laterally spreading tumor.

$\mathrm{CRC}=$ colorectal carcinoma.

a

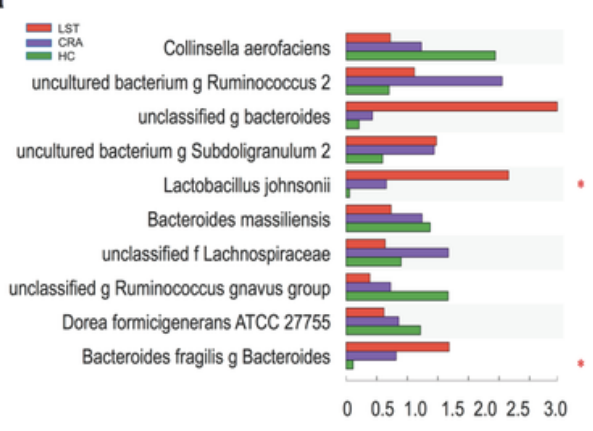

b

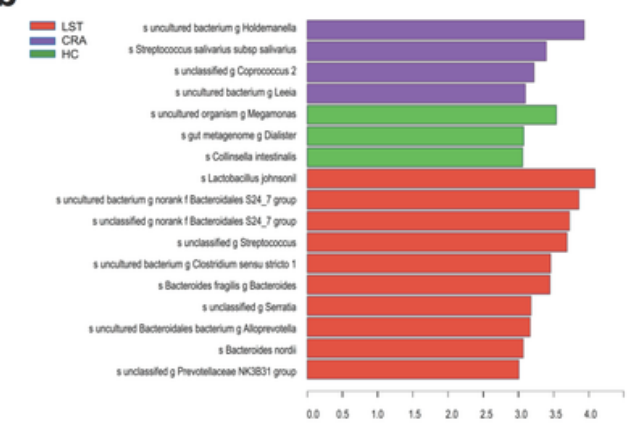

C

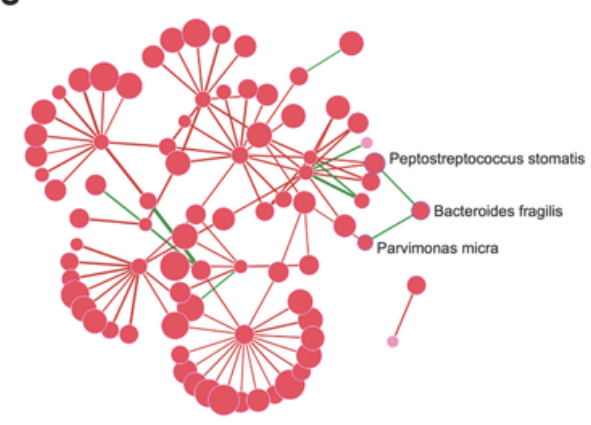

\section{Figure 3}

Variations of mucosal microbiota composition on species level in LST. a Kruskal-Wallis test analysis on species level in the three groups, Lactobacillus johnsonii and Bacteroides fragilis presented high abundance in the LST group. b LDA analysis on species level in the three groups, Lactobacillus johnsonii and Bacteroides fragilis significantly over-presented in the LST group. c The abundance of Bacteroides fragilis was positively related with the abundance of Peptostreptococcus stomatis and Parvimonas micra. $\mathrm{LDA}=$ linear discriminant analysis. $\mathrm{HC}=$ healthy control. $\mathrm{CRA}=$ colorectal adenoma. $\mathrm{LST}=$ laterally spreading tumor. 
a
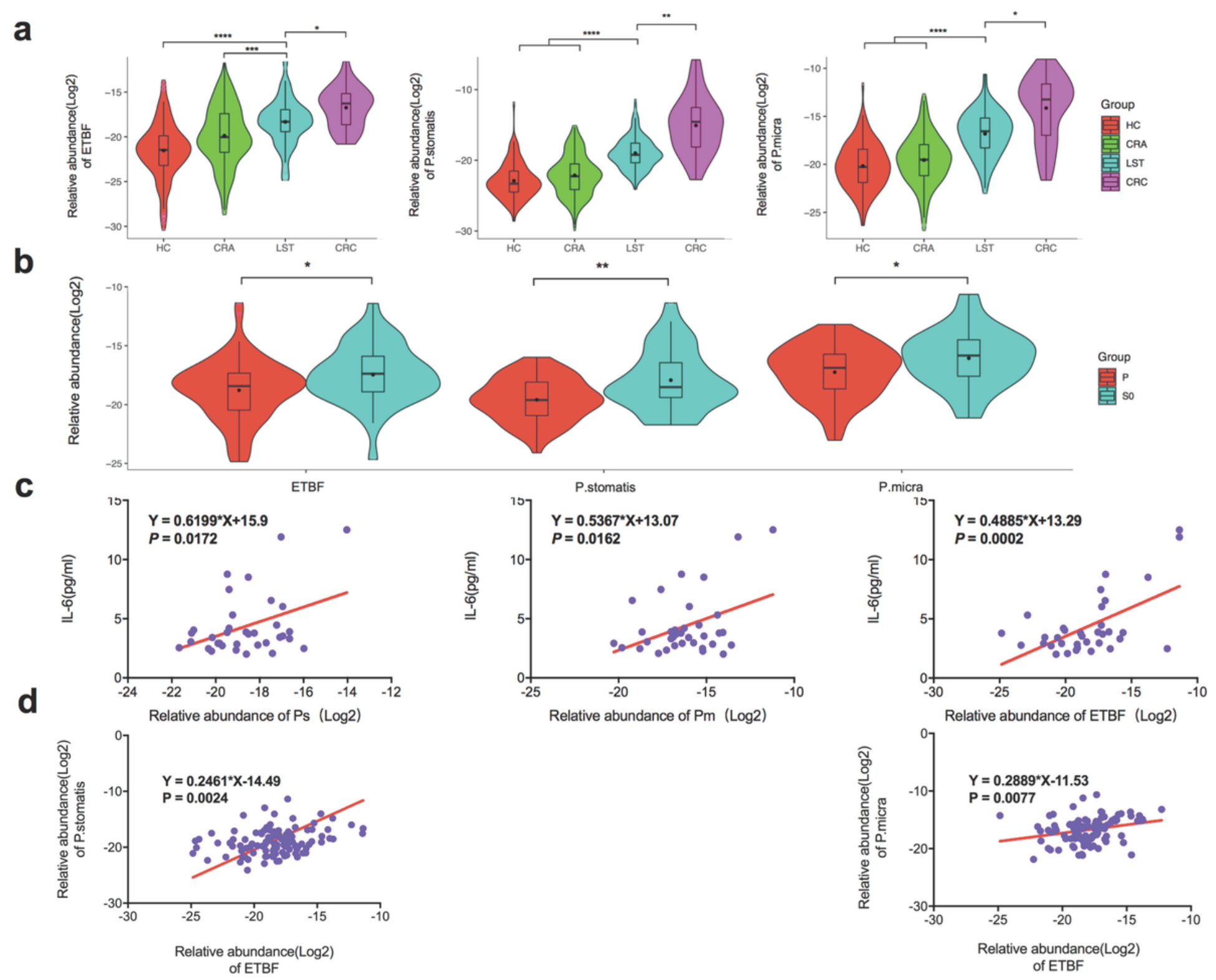

Figure 4

The fecal relative abundance of ETBF, P. stomatis and P. micra among groups. a The fecal relative abundance of ETBF, P. stomatis and P. micra was stepwise increased in the three groups. $b$ The fecal high abundance of ETBF, P. stomatis and P. micra was relevant with S0 stage LSTs. c The expression of IL-6 was positively related with the abundance of the three bacteria. $d$ The abundance of P.stomatis and P.micra increased with the increasing abundance of ETBF.HC=healthy control. CRA=colorectal adenoma. LST=laterally spreading tumor. $\mathrm{CRC}=$ colorectal carcinoma. $\mathrm{ETBF}=$ Enterotoxigenic Bacteroides fragilis. $\mathrm{P}$. stomatis $=$ Peptostreptococcus stomatis. $\mathrm{P}$. micra $=$ Parvimonas micra. $\mathrm{P}=\mathrm{LST}$ s with hyperplastic type or low-grade dysplasia. S0= LSTs with intramucosal carcinoma or high-grade dysplasia. ${ }^{*} P<0.05 .{ }^{* \star P}<0.01$. ${ }^{\star} * \star * P<0.001 .{ }^{*} * \star * P<0.0001$. 
a

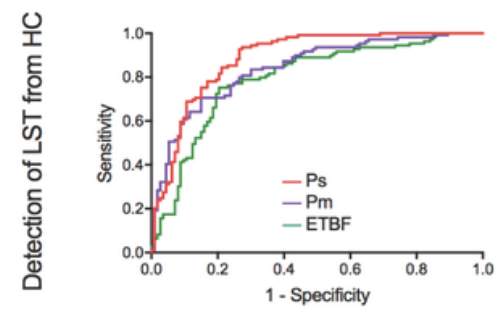

c

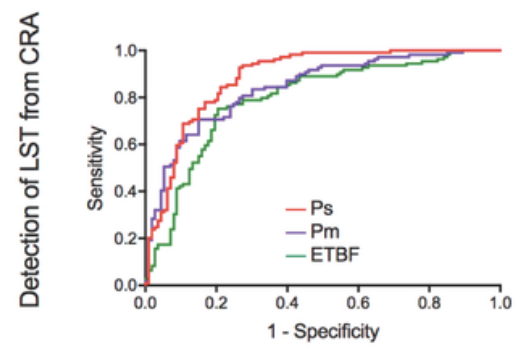

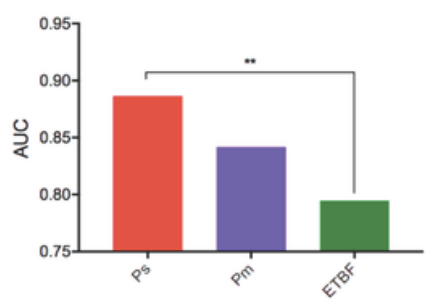

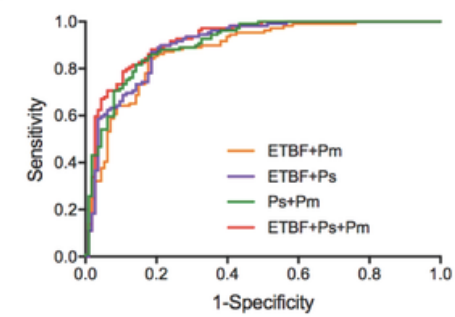

d
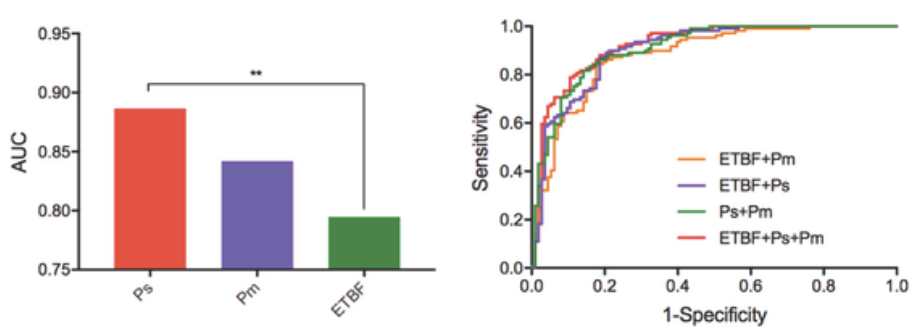

b
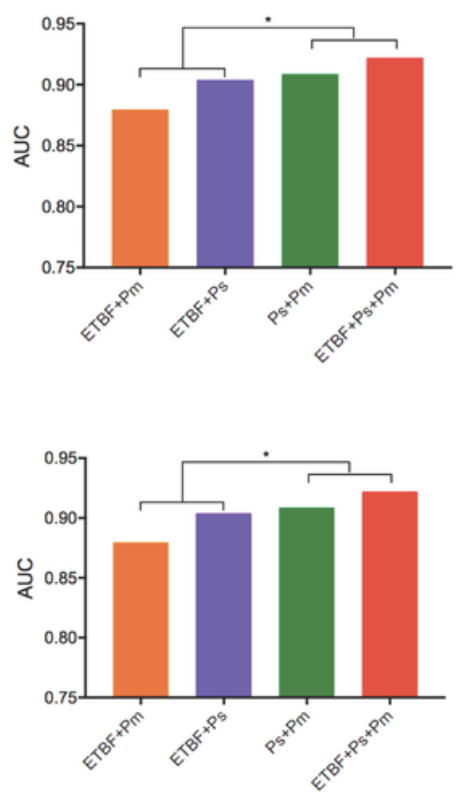

Figure 5

The diagnostic performance of marker ETBF, P. stomatis, P. micra and their combined test indicated by the receiver operating characteristic (ROC) curve analysis a Differentiating LST from HC, the ROC curves and AUCs (Delong's test) of the three bacteria. b Differentiating LST from HC, the ROC curves and the AUCs (Delong's test) of the promising diagnostic bacterial models. c Differentiating LST from CRA, the ROC curves and AUCs (Delong's test) of the three bacteria. d Differentiating LST from CRA, the ROC curves and the AUCs (Delong's test) of the promising diagnostic bacterial models. $\mathrm{HC}=$ healthy control. LST=laterally spreading tumor. ETBF = Enterotoxigenic Bacteroides fragilis.Ps= Peptostreptococcus stomatis. $\mathrm{Pm}=$ Parvimonas micra. $\mathrm{AUC}=$ area under the receiver-operating characteristic curve. ${ }^{*} \mathrm{P}<0.05$. $\star * P<0.01$. 


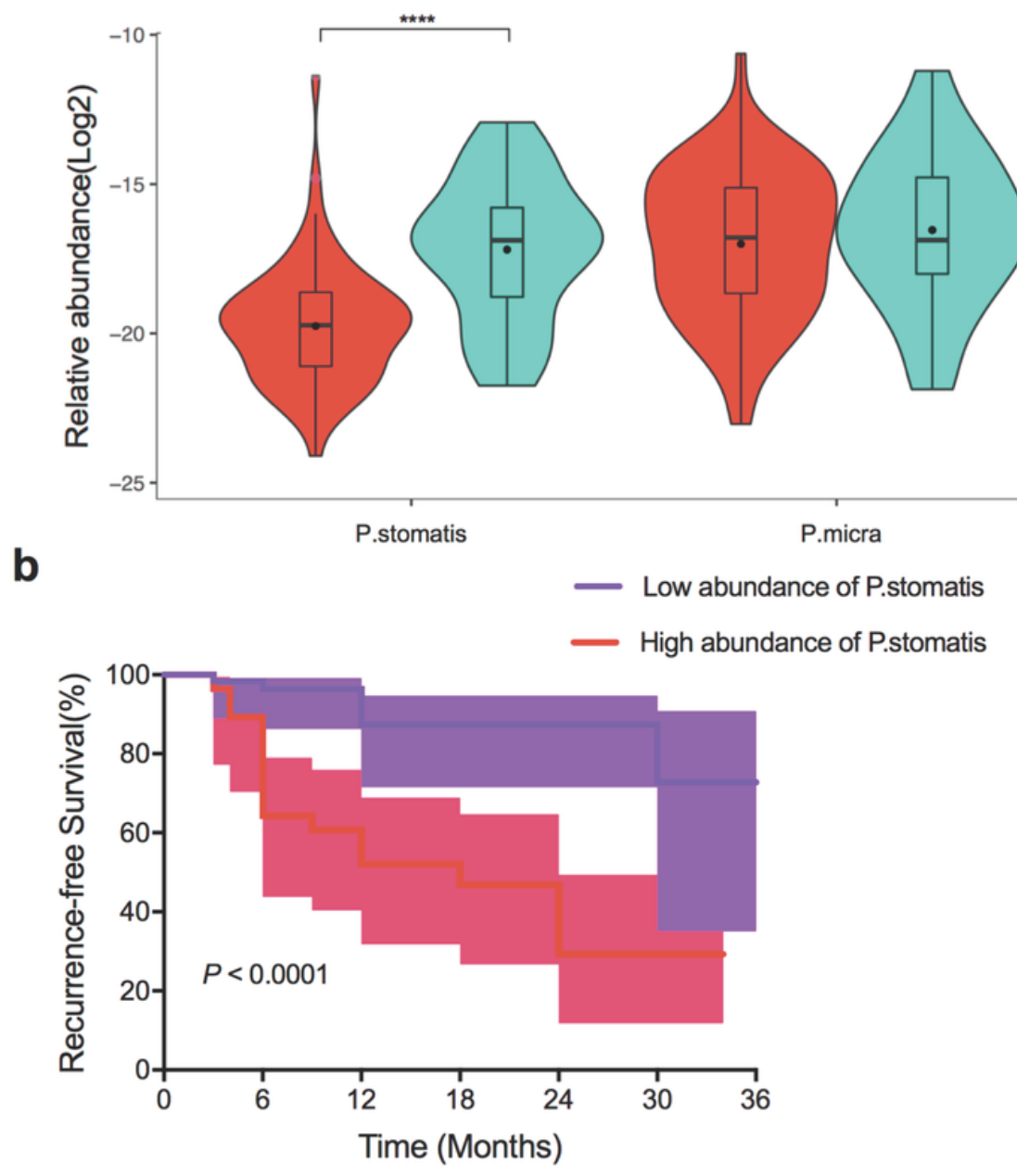

\section{Figure 6}

Performance of ETBF, P. stomatis and P. micra on adenoma recurrence after resection of LST. a The fecal relative abundance of P. stomatis, P. micra and ETBF among non-recurrence group and recurrence group. P. stomatis was related with adenoma recurrence. b Kaplan-Meier estimates for survival probability based on the abundance levels of P. stomatis in LST and histopathology of LST. High abundance of P. stomatis and S0 stage LST indicted adenoma recurrence. P. stomatis= Peptostreptococcus stomatis. P. micra= Parvimonas micra. ETBF = Enterotoxigenic Bacteroides fragilis. $\mathrm{P}=\mathrm{LST}$ s with hyperplastic type or lowgrade dysplasia. S0= LSTs with intramucosal carcinoma or high-grade dysplasia. ${ }^{* \star \star *} \mathrm{P}<0.0001$.

\section{Supplementary Files}

This is a list of supplementary files associated with this preprint. Click to download.

- SupplementaryFigure2.pdf

- SupplementaryFigure3.pdf

- SupplementaryFigure1.pdf 
- SupplementaryTables.docx 